to elucidate earthquake occurrence ${ }^{12}$ is, sadly and perhaps shamefully, rare. MuirWood and D. Giardini (Univ. Roma) remonstrated with the profession for devoting so few resources to seeking and husbanding our trove of historical earthquakes. These documents bear strongly on the issues of characteristic earthquakes, clustering and blind events, and because teasing science from diaries and church archives demands skills that few today possess, the historical treasure may be far more perishable than the strata in a trench.

Ross S. Stein is with the US Geological Survey, 345 Middlefield Road, Menlo Park, California 94025, USA.

\title{
Ordering chaos with disorder
}

\section{Steven H. Strogatz}

SYNCHRONIZATION of an array of nonlinear oscillators - for instance, coupled Josephson junctions or semiconductor lasers - is most easily achieved if all the oscillators are identical. Or so one might have thought. On page 465 of this issue, Braiman, Lindner and Ditto provide an intriguing counterexample. They start with an array of periodically forced pendula that lapses into spatiotemporal chaos if the pendula are identical. Yet when disorder is introduced into the array, the system snaps into periodic behaviour. In a sense, chaos has been tamed by disorder.

Oscillator arrays and spatiotemporal chaos are both relatively recent themes in nonlinear dynamics. In the late 1970 s and throughout the 1980 s, theorists focused on systems with few degrees of freedom. This was the heyday of the single damped driven pendulum, the Lorenz equations, and other small systems of ordinary differential equations, along with the logistic map, the Hénon map, and other simple iterated mappings. The key discovery from this era, due to Feigenbaum and others, was that there are universal laws governing the transition from periodic to chaotic behaviour: roughly speaking, diverse physical systems can go chaotic in the same way. This universality was beautifully explained by renormalization group ideas borrowed from statistical mechanics, and was later confirmed in experiments on fluids, electronic circuits, oscillating chemical reactions, semiconductors and mechanical systems (see, for example, P. Cvitanovic (ed.) Universality in Chaos 2nd edn; Hilger, Bristol, 1989).

Some of these successes seemed too good to be true. How could a spatially extended system, such as a fluid undergoing thermal convection, be governed by laws derived from simple mappings that contained none of the physics? The catch is that all the successful experimental tests were done on highly dissipative, spatially constrained systems. By design, these systems acted as though they had only a few degrees of freedom, so they naturally obeyed universality theory.

The outstanding problem today (and it will be with us for decades to come) is to develop techniques for understanding systems with many degrees of freedom, such as turbulent fluids or fibrillating hearts. As a first step, one approach is to investigate idealized caricatures of such complex
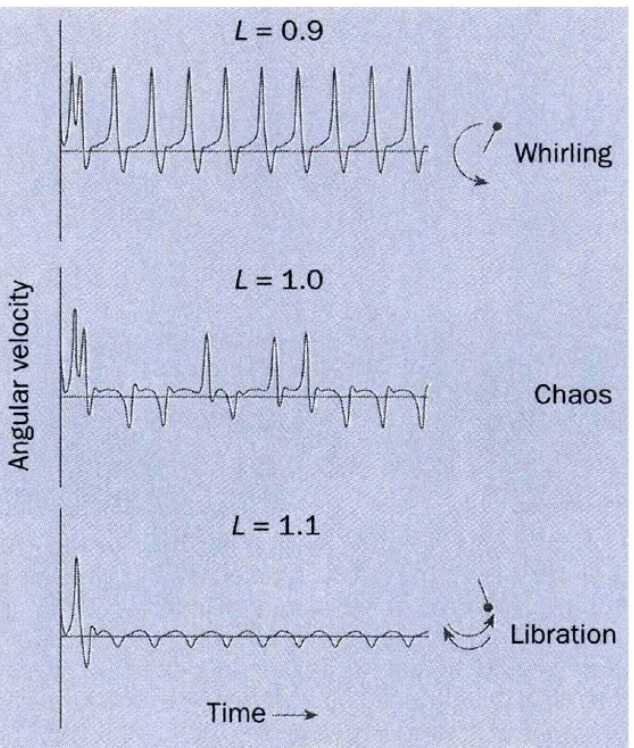

The motion of a single periodically driven pendulum depends on its length $L$. Both short and long pendula settle into periodic motions, either whirling (top panel) or libration (bottom panel), whereas a pendulum of intermediate length displays a chaotic sequence of whirling and libration.

systems. It is in this spirit that Braiman $e t$ al. have turned their attention to arrays of nonlinear oscillators.

Each of their oscillators is an underdamped pendulum driven periodically by a combined d.c. and a.c. torque (the net torque is always positive, but it varies sinusoidally). In response to such a drive, a single pendulum exhibits three types of long-term motion, depending on its length (see figure). For the parameters used by Braiman et al., a pendulum of dimensionless length 0.9 rapidly settles into a periodic whirling motion, completing exactly one full rotation for each cycle of the dri- ving torque. Frequency-locking to the a.c. part of the drive also occurs if the pendulum has length 1.1, but now the pendulum hangs downwards at all times, librating once about the downward vertical per drive cycle. For intermediate length 1.0, the pendulum's motion is chaotic; irregular librations are sporadically interrupted by rotations over the top.

Braiman et al. consider a chain of such pendula coupled to their nearest neighbours by torsional springs. In a homogenous chain where all the pendula have length 1.0 (as in Fig. 1a of Braiman et al.), the chaos of the individual oscillators persists in the coupled array; all the pendula whirl and librate, but with no repeating spatial or temporal structure. However, if the lengths of the pendula are randomly distributed across the chain, say with a 10 or 20 per cent uniform spread, the array breaks up into well defined domains that are frequency-locked (at various ratios) to the a.c. drive. An explanation given by Braiman et al. seems entirely plausible, in light of our discussion - the disorder creates subpopulations of long and short pendula that can easily frequency-lock to the a.c. drive. It does not matter whether these locked pendula are librating or whirling; they impose their rhythm, via the coupling, on those that would otherwise be chaotic, thus taming the chaos in the entire array.

A much more dramatic effect would be to tame chaos through disorder while keeping all the pendula in their chaotic regimes. Braiman et al. claim that they have observed this too, in which case a more subtle mechanism must also be at play.

Future work should test whether disorder-induced taming is common in spatially extended systems, and quantify the likelihood of its occurrence. Regarding potential applications, Braiman et al. argue that disorder might be used to 'control' spatiotemporal chaos, but that seems optimistic, given that no one knows what complex periodic pattern will arise after disorder is introduced. Nevertheless, taming might still be useful in mode-locking applications where any kind of periodic behaviour is preferred to chaos. For instance, superconducting Josephson arrays or semiconductor laser arrays are two systems in which collective locking is technologically desirable, but notoriously difficult to achieve. Normally the difficulty is blamed on the inevitable inhomogeneities, but now we have to wonder whether better results might be achieved by disordering the systems even further. Admittedly, the result will not be coherent oscillation, but perhaps a complex spatial pattern of frequency-locking is better than no locking at all.

Steven H. Strogatz is in the Department of Theoretical and Applied Mechanics and the Center for Applied Mathematics, Cornell University, Ithaca, New York 14853, USA. 